\title{
NBTI prediction and its induced time dependent variation
}

\author{
Jian F. Zhang*, Meng Duan, Zhigang Ji, and Weidong Zhang \\ School of Engineering, Liverpool John Moores University, Liverpool L3 3AF, UK \\ *Email: j.f.zhang@ljmu.ac.uk
}

\begin{abstract}
Negative bias temperature instability (NBTI) prediction relies on a reliable extraction of power exponents from its kinetics. When measured by fast pulse technique, however, the kinetics does not follow a power law. This paper reviews the recent progresses on how to restore the power law, based on the $\underline{A}$ s-grown-Generation (AG) model. For nanometer sized devices, NBTI is different for different devices, inducing a time-dependent variation. The new technique proposed for characterizing this Time-dependent $\underline{\text { Variation accounting for }}$ within-a-device-Fluctuation (TVF) will be reviewed.
\end{abstract}

\section{Introduction}

Conventionally, NBTI aging follows a power law [1,2] and the power exponent ' $n$ ' in eq.(1) does not change with stress voltage, Vgst,

$$
\Delta \mathrm{Vth}=\mathrm{gVgst}^{\mathrm{m}} \mathrm{t}^{\mathrm{n}}
$$

where $\Delta \mathrm{Vth}$ is the NBTI-induced threshold voltage shift, $\mathrm{g}$ the generation constant, and the stress time. When measured by quasi-DC parameter analyzer, Fig. 1(a) shows that data follows eq.(1) well. This is the foundation for reliable prediction of NBTI-induced device lifetime [2,3]. It is well known, however, NBTI recovers substantially during the quasi-DC measurement $[4,5]$. When the recovery is suppressed by using the fast pulse technique, Fig. 1(b) shows that NBTI no longer follows eq.(1) [2]. To achieve reliable prediction in this case, an As-grown-Generation (AG) model has been proposed, based on the framework for positive charges in gate dielectric [6-18].

\section{As-grown-Generation (AG) model}

The positive charges in gate dielectric can be divided into two groups: as-grown hole traps (AHTs) and generated defects (GDs) [6-18]. AHTs are located below Si valence band edge, Ev, while GDs are above Ev [6,7], as shown in Fig. 2. The energy profile in Fig. 2 was obtained by using the discharge-based technique [8]. Their different energy locations allow AHTs being separated from GDs. Fig. 3 shows that NBTI is dominated by filling AHTs initially, while the generated defects control the aging for longer time [2,3,9]. Once all AHTs are filled, they saturates, but the GDs follow the power law without saturation [2,3]. Filling AHTs and generating GDs are two independent processes [10-14]. This understanding has led to the As-grown-Generation (AG) model,

$$
\Delta \mathrm{Vth}=\mathrm{A}+\mathrm{gVgst}^{\mathrm{m}} \mathrm{t}^{\mathrm{n}}
$$

where 'A' represents the saturation level of AHTs. After removing the ' $A$ ', the power law is restored for the GDs, as shown in Fig. 4, allowing reliable NBTI prediction.
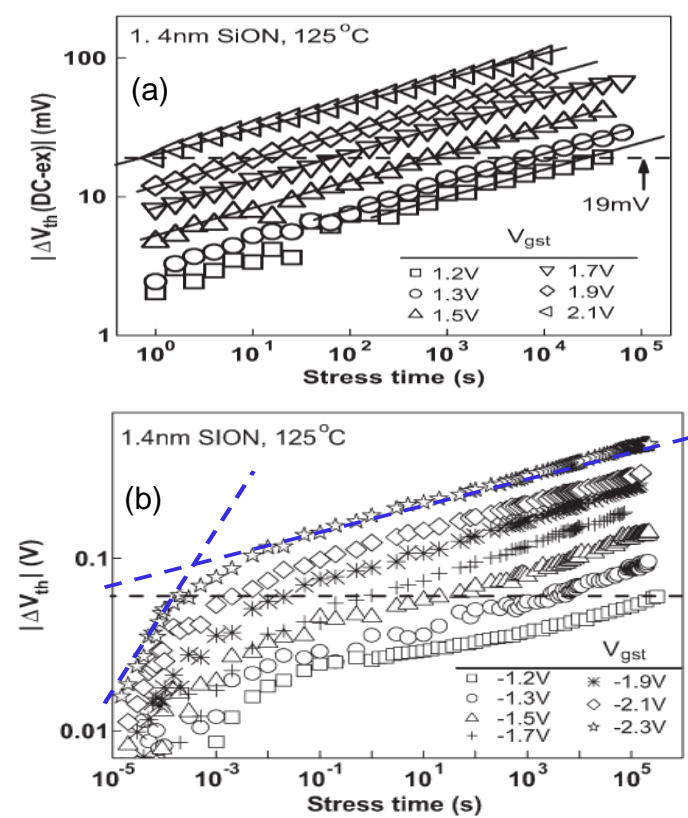

Fig. 1. NBTI follows the power law when measured by quasi-DC method (a), but does not when measured by pulse $(5 \mu \mathrm{s})$ technique (b) [2].

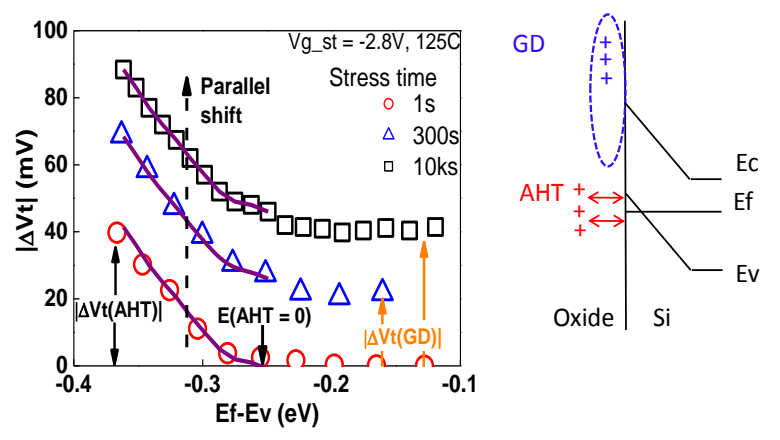

Fig. 2. AHTs and GDs are below and above Si Ev, respectively. [3] 


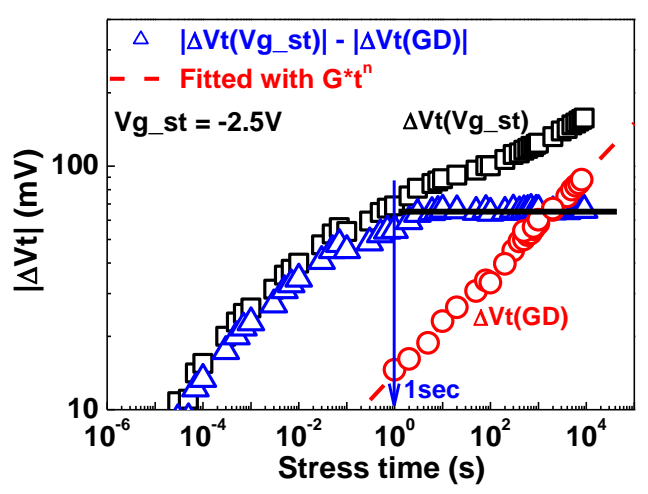

Fig. 3. AHTs $(\Delta)$ saturate and GDs (o) follow power law [3].

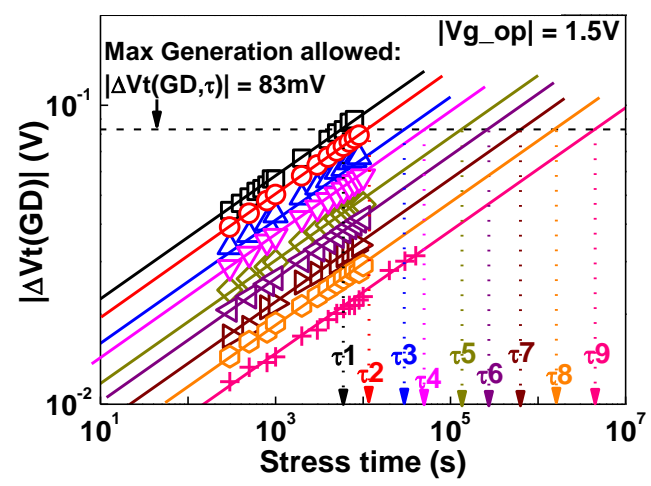

Fig. 4. After removing AHTs, GDs follow power law well with thw power exponents independent of stress voltage [3].

\section{Within a device fluctuation (WDF)}

For nanometer devices, individual-charging/discharging can be observed and Fig.5 shows that NBTI-induced aging at a given stress time is no longer a fixed value [19-22]. It consists of two components: a winthin-a-device fluctuation (WDF) and a lower-envelop (LE) that does not discharge [19-22]. The upper panel in Fig. 5 shows that the WDF within a short time window has the signature of random telegraph noises (RTN).

To capture the WDF, the impacts of sampling rate and time window are investigated. Fig. 6 shows that WDF increases with sampling rate initially, as higher rate allows capturing faster traps. When the sampling rate reaches $1 \mathrm{MS} / \mathrm{sec}$, WDF saturates, as all traps are captured. A sampling rate of $10 \mathrm{MS} / \mathrm{sec}$ will be used hereafter [19,20].

When plotted in linear scale, Fig. 7 shows that the WDF appears insensitive to time window. This is, however, an artefact. After plotting the time in logarithmic scale, it is clear that WDF increases for larger time window. As a result, the time window used for capturing WDF should be as large as practically possible $[19,20]$.

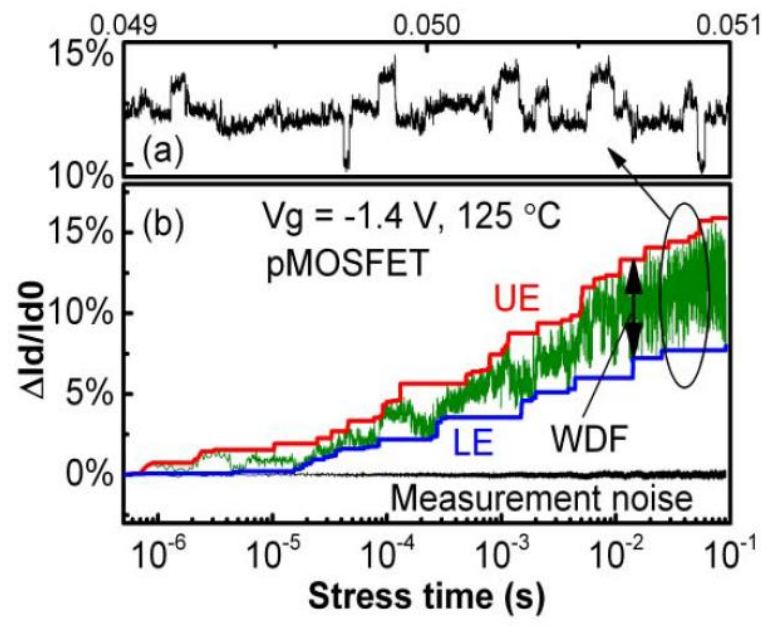

Fig. 5. (a) For a given defect number, the RTN signal by en-larging the circled region of (b). Defects, however, increase with time. The 'UE' and 'LE' represent the upper and lower envelope of raw data and their difference is caused by charge fluctuation under a given $\mathrm{Vg}$ [20].
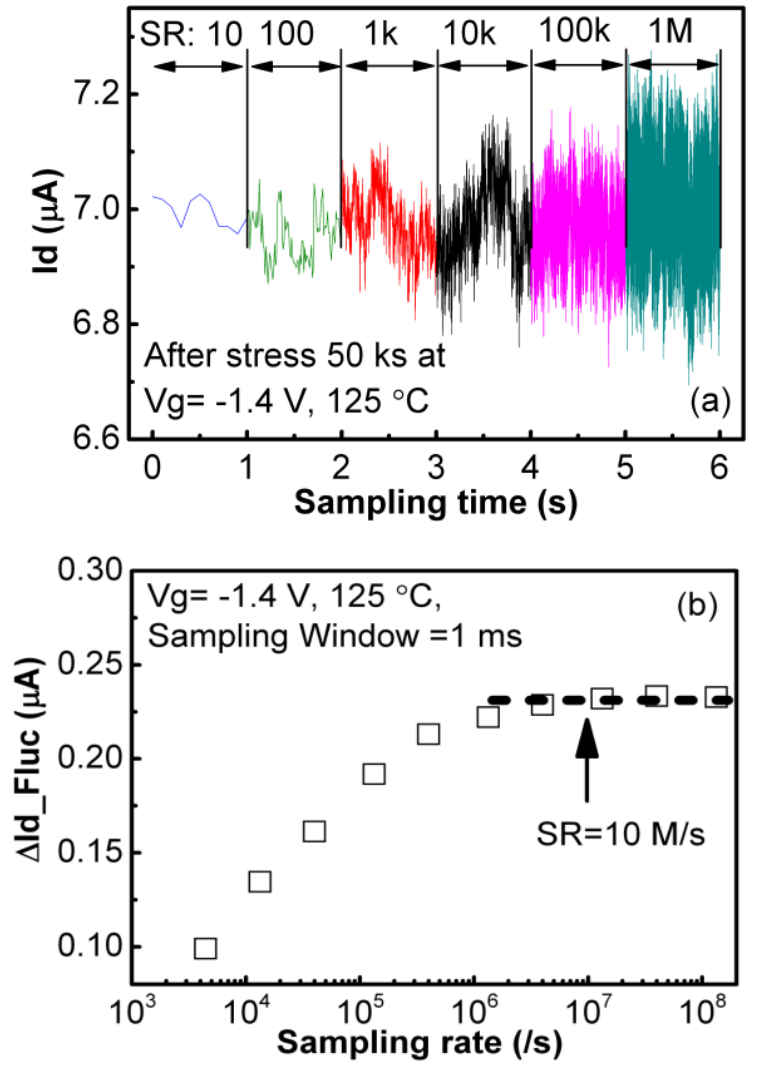

Fig. 6. Id fluctuation increases with sampling rate (SR) when $\mathrm{SR}<1 \mathrm{M} / \mathrm{s}$ (a), but saturates after $\mathrm{SR}>1 \mathrm{M} / \mathrm{s}$ (b) [20]. 

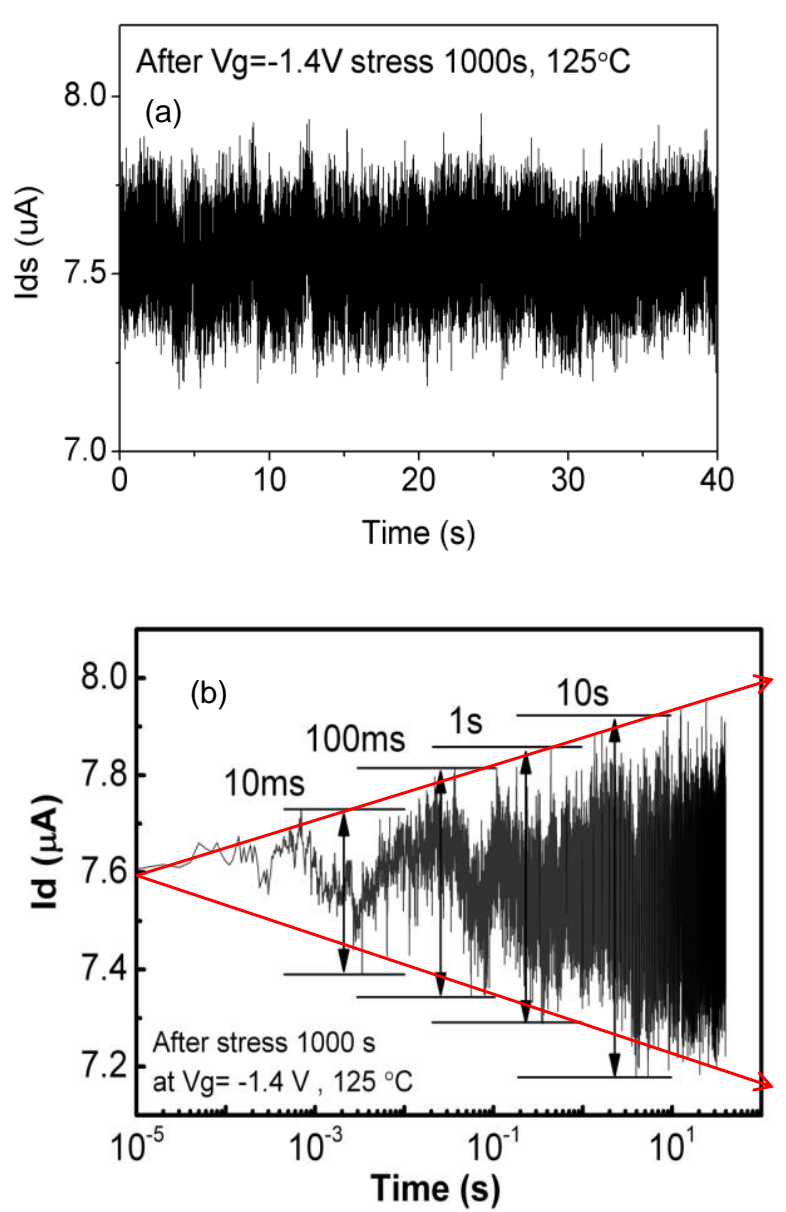

Fig. 7 Dependence of WDF on the measurement time window. Although the Id fluctuation appears insensitive to time in a linear scale in (a), it clearly increases with time when plotted in a logarithmic scale in (b) [20].

\section{Time-dependent device-to-device variation}

The presence of WDF means that $\Delta \mathrm{Vth}$ measured twice can give different values. The question is which value should be used when comparing with other devices. To answer it, a Time-dependent Variation taking into account of the Fluctuation (TVF) technique has been proposed [17]. Central to TVF is that when comparing different devices, one must not use the instantaneous $\Delta V$ th at a time point. Instead, the lower envelop (LE) in Fig. 5 and its difference from the upper-envelop (UE), i.e. WDF=UE-LE, should be used. One example for the device-to-device variation of LE is given in Fig. 8.

Fig. 9(a) shows the average values of UE, LE, and WDF for 52 devices. Both LE and WDF are important. The average value of LE can be predicted by the AG model [18].

The standard deviations of UE, LE and WDF are given in Fig. 9(b). As stress increases, both the average and standard variation increases. Fig. 10 shows that the standard deviation follows a power law against the average value. As a result, once the average value is predicted, the standard deviation can be determined from such relation [18].

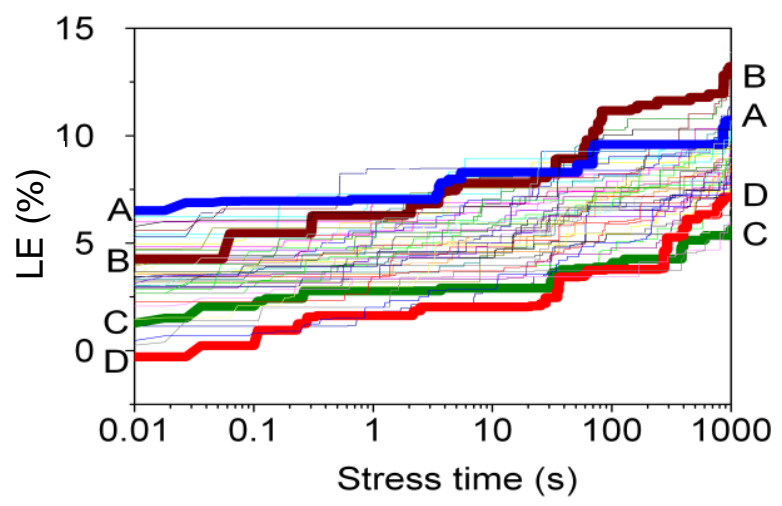

Fig. 8. Device-to-device variations of the lower-envelope, LE. The thick curves marked by 'A, $\mathrm{B}, \mathrm{C}, \mathrm{D}$ ' are the results of boundary devices [21].
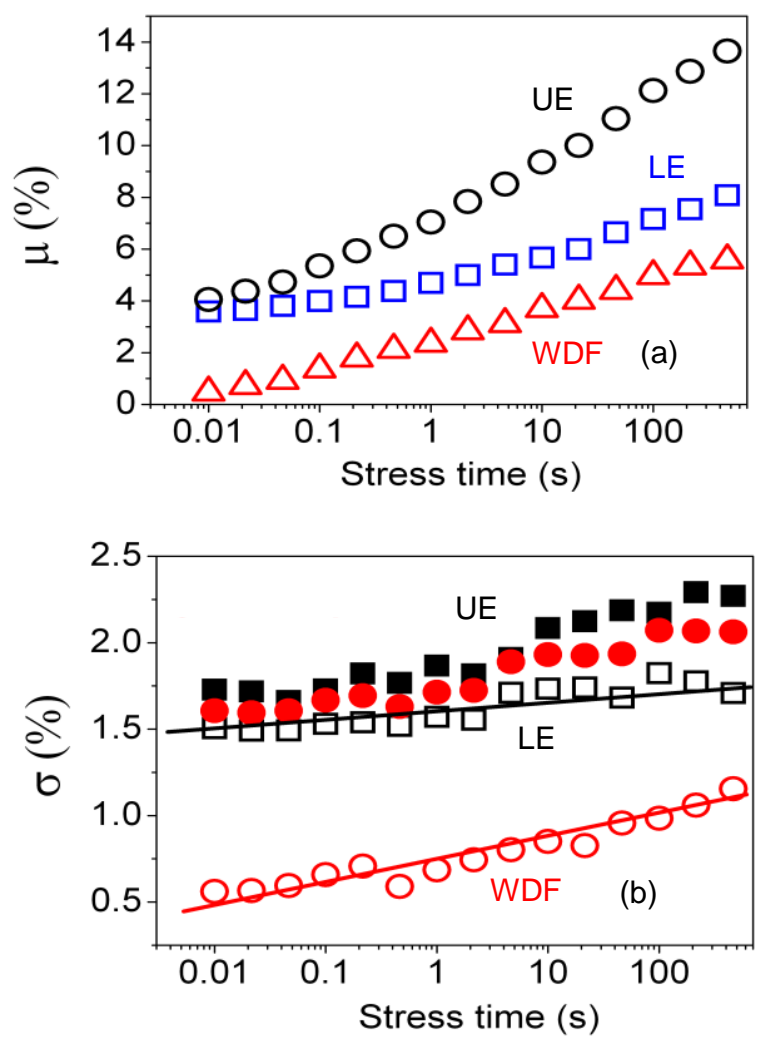

Fig. 9. (a) and (b) compare UE, LE and WDF for the average and standard deviation, respectively. The symbol ' $\bullet$ ' is the calculated value [21]. 


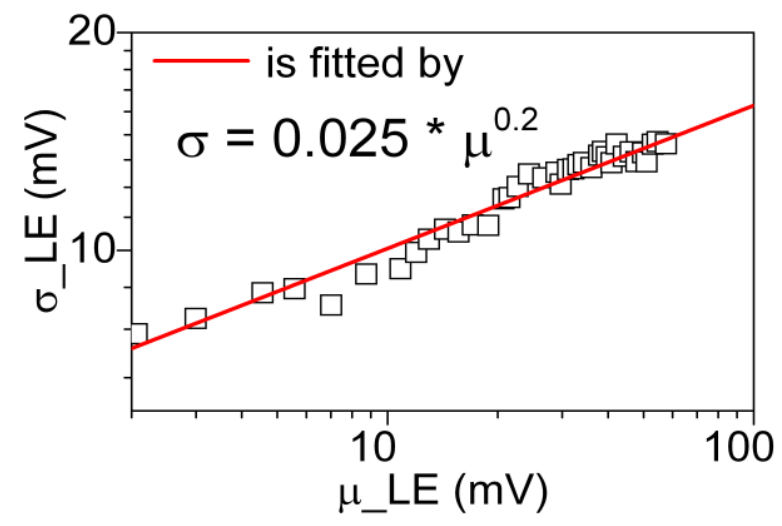

Fig. 10. The relationship between the standard deviation and the average for LE [22].

\section{Conclusions}

This work reviews the recent development in the NBTI modelling and prediction. The positive charges responsible for NBTI can be divided into as-grown hole traps (AHTs) and generated defects (GDs). AHTs are below the silicon valence band edge, dominates NBTI initially, and saturates for longer stress time. In contrast, GDs are above silicon valence band edge and follow a power law without saturation. This AG model restores the power law, allowing the long term NBTI prediction. For nanometer devices, the Time-dependent Variation accounting for within-a-device-Fluctuation (TVF) technique has been developed to separate the fluctuation from the real device-to-device variation.

\section{Acknowledgments}

The authors would like to thank B. Kaczer and G. Groeseneken of IMEC, Belgium, for supply of test samples and discussions. This work is supported by EPSRC of UK under the grant no. EP/L010607/1.

\section{References}

[1] K. O. Jeppson and C. M. Svensson, J. Appl. Phys., 48, p.2004 (1977).

[2] Z. Ji, L. Lin, J. F. Zhang, B. Kaczer, and G. Groeseneken, IEEE Trans. Electron Dev., 57, p. 228 (2010).

[3] Z. Ji, S. F. W. M. Hatta, J. F. Zhang, J. G. Ma, W. Zhang, N. Soin, B. Kaczer, S. De Gendt, and G. Groeseneken, IEDM, p. 413 (2013).

[4] J. F. Zhang, Z. Ji, M. H. Chang, B. Kaczer, and G. Groeseneken, IEDM, p.817 (2007).

[5] Z. Ji, J. F. Zhang, M. H. Chang, B. Kaczer, and G. Groeseneken, IEEE Trans. Electron Dev., 56, p. 1086 (2009).

[6] C. Z. Zhao, J. F. Zhang, M. H. Chang, A. R. Peaker, S. Hall, G. Groeseneken, L. Pantisano, S. De
Gendt, and M. Heyns, IEEE Trans. Electron Devices, 55, p. 1647 (2008).

[7] J. F. Zhang, Microelectron. Eng., 86, p. 1883, (2009).

[8] S. F. Wan Muhamad Hatta, Z. Ji, J. F. Zhang, M. Duan, W. Zhang, N. Soin, B. Kaczer, S. De Gendt, and G. Groeseneken, IEEE Trans. Electron Dev., 60, p. 1745 (2013).

[9] J. F. Zhang, H. K. Sii, G. Groeseneken, and R. Degraeve, "Hole trapping and trap generation in the gate silicon dioxide," IEEE Trans. Electron Devices, vol. 48, no. 6, pp. 1127, Jun, 2001.

[10] J. F. Zhang, C. Z. Zhao, A. H. Chen, G. Groeseneken, and R. Degraeve, IEEE Trans. Electron Devices, 51, p. 1267 (2004).

[11] C. Z. Zhao, J. F. Zhang, G. Groeseneken, and R. Degraeve, IEEE Trans. Electron Devices, 51, p.1274 ( 2004).

[12] C. Z. Zhao, and J. F. Zhang, J. Appl. Phys., 97, art.no. 073703 (2005).

[13] M. H. Chang, and J. F. Zhang, J. Appl. Phys., 101, art.no.024516 (2007).

[14] J. F. Zhang, M. H. Chang, and G. Groeseneken, IEEE Electron Dev. Lett., 28, p.298 (2007).

[15] J. F. Zhang, M. H. Chang, Z. Ji, L. Lin, I. Ferain, G. Groeseneken, L. Pantisano, S. De Gendt, and M. M. Heyns, IEEE Electron Device Letters, 29, p.1360 (2008).

[16] C. Z. Zhao, J. F. Zhang, M. B. Zahid, G. Groeseneken, R. Degraeve, and S. De Gendt, Appl. Phys. Lett., 89, art.no. 023507 (2006).

[17] C. Z. Zhao, M. B. Zahid, J. F. Zhang, G. Groeseneken, R. Degraeve, and S. De Gendt, Appl. Phys. Lett., 90, art.no.143502 (2007).

[18] . J. F. Zhang, C. Z. Zhao, M. H. Chang, M. B. Zahid, A. R. Peaker, S. Hall, G. Groeseneken, L. Pantisano, S. De Gendt, and M.Heyns, Appl. Phys. Lett., 92, art.no. 013502 (2008).

[19] . M. Duan, J. F. Zhang, Z. Ji, J. G. Ma, W. Zhang, B. Kaczer, T. Schram, R. Ritzenthaler, G. Groeseneken, and A. Asenov, IEDM, p. 774 (2013).

[20] M. Duan, J. F. Zhang, Z. Ji, W. D. Zhang, B. Kaczer, T. Schram, R. Ritzenthaler, G. Groeseneken, and A. Asenov, IEEE Trans. Electron Dev., 61, p. 3081 (2014).

[21] M. Duan, J. F. Zhang, Z. Ji, W. Zhang, B. Kaczer, T. Schram, R. Ritzenthaler, G. Groeseneken, and A. Asenov, IEEE Trans. Electron Dev., 60, p.2505 ( 2013).

[22] M. Duan, J. F. Zhang, Z. Ji, W. Zhang, B. Kaczer, T. Schram, R. Ritzenthaler, A. Thean, G. Groeseneken, and A. Asenov, VLSI Tech. Symp., p.74 (2014). 